\title{
Parity effect in mesoscopic and nanoscopic superconducting particles
}

\author{
I.O. Kulik *, H. Boyaci, Z. Gedik \\ Department of Physics, Bilkent University, Bilkent 06533, Ankara, Turkey
}

\begin{abstract}
Superconductivity in small metallic specimens is studied with regard to the size dependence of the parity gap $\left(\Delta_{\mathrm{P}}\right)$, a parameter distinguishing between the energy of even and odd number of electrons in the granule. $\Delta_{\mathrm{P}}$ is shown to be an increasing function of level spacing $\delta$. The energy gap of superconductor $\Delta$, on the other hand, decreases with increasing $\delta$ and vanishes at $\delta=\delta_{\mathrm{c}}$ which is of the order of $\Delta$. However, non-zero value of $\Delta_{\mathrm{P}}$ persists above $\delta_{\mathrm{c}}$ in a gapless superconducting-insulating state. Level degeneracy in small specimens having perfect geometry changes the size dependence of the parity gap, the Josephson effect, and flux quantization. Parity gap is evaluated using an interpolation procedure between the continuum limit $(\delta \ll \Delta)$, the moderate mesoscopic regime $(\delta \sim \Delta)$, and the nanoscopic scale $(\delta \gg \Delta)$, for which an exact solution to the pairing problem is provided with the numeric diagonalization of system Hamiltonian in a small metallic cluster. (c) 2001 Elsevier Science B.V. All rights reserved.
\end{abstract}

Keywords: Mesoscopic superconductivity; Parity effect; Parity gap; Strongly correlated systems; Pseudogap; Gapless state

\section{Introduction}

Superconductivity in metals is related to condensation of electrons into pairs which are extended objects with a size of order $\xi \sim \hbar v_{\mathrm{F}} / \Delta_{0}$. A fundamental question [1] is whether pairing can survive in small specimens with size $d$ less than $\xi$. In energy scale this corresponds to condition that $\delta_{1}>\Delta_{0}$ where $\delta_{1}=\hbar v_{\mathrm{F}} / d$ is typical scale of dimensional energy quantization. The energy levels can remain highly degenerate in a perfect sample (see Fig. 1) but in a disordered sample, level spacing $\delta_{2} \sim \varepsilon_{\mathrm{F}} / N_{\mathrm{s}}$, where $N_{\mathrm{s}}$ is the total number of

\footnotetext{
* Corresponding author. Fax: +90-312-2664579.

E-mail address: kulik@fen.bilkent.edu.tr (I.O. Kulik).
}

sites (which is of the order of total number of electrons, $N$ ), is much smaller than $\delta_{1}$ and can put a limit to superconducting pairing at $\delta_{2} \sim \Delta_{0}$. In recent experiments [2,3], signature of superconducting pairing was traced in ultrasmall specimens down to sizes of $10 \mathrm{~nm}$. The superconductivity reveals itself through the number-parity effect, the dependence of superconducting energy on whether the number of electrons in sample is even or odd. Matveev and Larkin [4] introduced a parity gap $\Delta_{\mathrm{P}}=(1 / 2)\left(E_{2 n+1}+E_{2 n-1}\right)-E_{2 n}$ where $E_{n}$ is superconducting energy vs number of electrons $n$, to characterize that the paired state is preferable over the state with odd number of electrons. In a number of papers, in particular in the paper of Braun and von Delft [5], $E_{n}$ was calculated assuming that in an odd-number sample, electron 


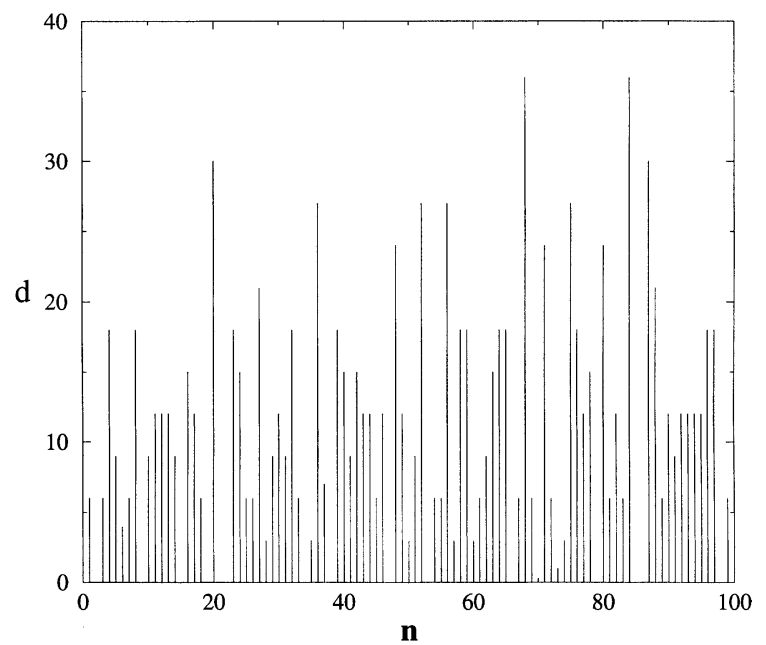

Fig. 1. Degeneracy of energy levels for parabolic energy vs momentum dependence of total energy $E_{n}=\mathrm{Cn}^{2}=$ $C\left(n_{1}^{2}+n_{2}^{2}+n_{3}^{2}\right)$.

state near the Fermi energy is blocked from pairing, similar to the way in which finite-number systems are treated in nuclear physics [6]. In present paper, we investigate the suppression of superconductivity in a finite size system by using an approximation similar to that of [5], and also in the opposite limit, by starting from atomic-scale system (small atomic cluster) for which an exact solution of pairing problem is provided, and trying to match small- and large-size approximations through an interpolation procedure.

\section{Large-scale approximation}

In the Bardeen-Cooper-Schrieffer (BCS) approximation [7] a variational density matrix $\rho_{\alpha \beta}=(1 / Z) \phi_{\alpha}^{*} \exp (-(H-\mu N) / k T) \phi_{\beta}$ is exploited to obtain the ground state energy with trial wave functions

$$
\begin{aligned}
& \phi_{\alpha}^{(1)}=\prod_{\mathbf{k}}\left(u_{\mathbf{k}}+v_{\mathbf{k}} a_{\mathbf{k} \uparrow}^{\dagger} a_{-\mathbf{k} \downarrow}^{\dagger}\right)|0\rangle, \\
& \phi_{\alpha}^{(2)}=\prod_{\mathbf{k}_{\mathrm{F}}}\left(u_{\mathbf{k}}+v_{\mathbf{k}} a_{\mathbf{k} \uparrow}^{\dagger} a_{-\mathbf{k} \downarrow}^{\dagger}\right) a_{\mathbf{k}_{\mathrm{F}}}^{\dagger}|0\rangle
\end{aligned}
$$

for even and odd number of electrons, respectively. In the BCS pairing scheme (case 1), assuming equally spaced levels with distance $\delta$, we receive $\Delta_{2 m}=\Delta_{2 m+1}=\Delta_{0}$ whereas in the blocked pairing state (case 2) $\Delta_{2 m}=\Delta_{0}$ and $\Delta_{2 m+1}=\Delta_{0}-\delta / 2$. The energy of superconductor at $T=0$ is calculated as $\langle H-\mu N\rangle=\sum_{n}\left(\xi_{n}-\left(\Delta^{2}+\xi_{n}^{2}\right)^{1 / 2}\right)$ resulting in a parity gap value $\Delta_{\mathrm{P}}^{(1)}=\delta / 4$ (for $\delta \ll \Delta_{0}$ ) in the first case, and $\Delta_{\mathrm{P}}^{(2)} \simeq \Delta_{0} \ln \left(\omega_{\mathrm{D}} \delta / \Delta_{0}^{2}\right)+(1 / 2) \delta$ in the second. Energy of the blocked state is larger than that of the BCS state and therefore there is no reason to believe that the former is a better approximation for pairing. There is no direct relation of the above calculation to the question that there is a large uncertainty in the number of particles, and assertion that number of particles in $\phi_{\alpha}^{(2)}$ is odd is merely an illusion.

\section{Josephson effect in mesoscopic metal particles}

Consider two identical small superconducting grains interacting through the Hamiltonian $H_{T}=$ $\sum_{n m} T_{n m} a_{n}^{\dagger} b_{m}+$ h.c. where $a_{n}^{\dagger}\left(a_{n}\right)$ creates (annihilates) an electron in the first grain in state $n$. Similarly $b_{n}^{\dagger}$ and $b_{n}$ are operators for the second grain. By introducing the phases $\phi_{1}$ and $\phi_{2}$ to the Bogoliubov $u, v$-coefficients in Eq. (1), we calculate coupling energy due to $H_{T}$ as a function of $\phi_{1}$ and $\phi_{2}$ in a standard way [7] $\Delta E=\Delta E_{\mathrm{S}}^{\prime}-$ $\Delta E_{\mathrm{S}} \cos \left(\phi_{1}-\phi_{2}\right)$ where

$$
\Delta E_{\mathrm{S}}=\left\langle|T|^{2}\right\rangle \Delta^{2} \sum_{\left|\xi_{n}\right|,\left|\xi_{m}\right|<\omega_{\mathrm{D}}} \frac{1}{\left(\varepsilon_{n}+\varepsilon_{m}\right) \varepsilon_{n} \varepsilon_{m}} .
$$

The stationary Josephson current at zero temperature is $J_{\mathrm{c}} \sin \left(\phi_{1}-\phi_{2}\right)$ where $J_{\mathrm{c}}=2 e \Delta E_{\mathrm{S}} / \hbar$. Fig. 2 shows dependence of Josephson critical current and energy gap as a function of gap suppression parameter $\delta / \Delta_{0}$. The curves show that superconductivity is suppressed at level spacing $\delta_{\mathrm{c}}=3.56 \Delta_{0}$. This however is a conclusion within the mean-field approximation. It does not exclude the possibility that pairing may survive for $\delta>\delta_{\mathrm{c}}$ as a gapless superconducting state or as a state of superconducting insulator with an effective insulator gap $\delta$ larger than the superconducting pseudogap. 


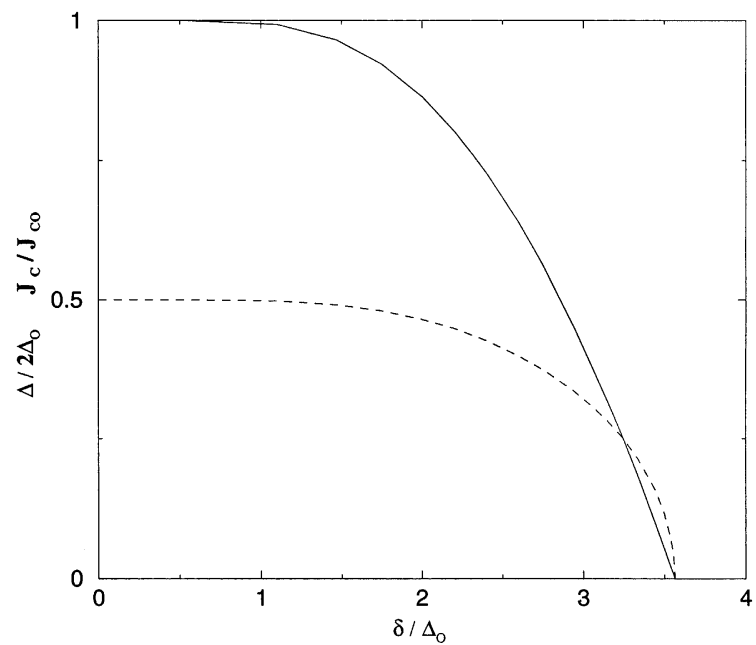

Fig. 2. Josephson critical current vs energy level spacing $\delta(-)$ and gap parameter $\Delta$ vs $\delta(---)$. The latter has been scaled by a factor of $1 / 2$ for convenience.

\section{Richardson-Sherman approximation}

A finite size solution to the pairing problem was suggested by Richardson and Sherman [8] (and recently revived by von Delft and Braun [9]) assuming that states with energy within the interac- tion shell $-\omega_{\mathrm{D}}<\xi_{n}<\omega_{\mathrm{D}}$ can be approximated as hard-core bosons whereas states away from this shell are treated exactly as non-interacting particles. The interaction Hamiltonian for hard-core bosons is

$H=2 \delta \sum_{n \alpha} n c_{n \alpha}^{\dagger} c_{n \alpha}-\frac{V \delta}{d} \sum_{\left|\check{\xi}_{n}\right|,\left|\xi_{m}\right|<\omega_{\mathrm{D}}, \alpha \beta} c_{n \alpha}^{\dagger} c_{m \beta}$

where boson operators are $c_{n \alpha}=a_{n \bar{\alpha}\rfloor} a_{n \alpha \uparrow}, d$ is level degeneracy, and $\alpha$ labels states within the degenerate energy level $\xi_{n}=n \delta$. Hamiltonian (3) is solved [10] by iteration scheme proposed by Richardson and Sherman [8]. Dependence of $\Delta_{\mathrm{P}}$ on $\delta / \Delta_{0}$ is shown in Fig. 3. In non-degenerate case $(d=1) \Delta_{\mathrm{P}}(\delta)$ shows a behavior similar to previous results with smaller number of levels [11,12]. In case of degenerate energy levels, pairing gap is multi-valued depending upon which three successive states are used in calculation of $\Delta_{\mathrm{P}}$. The true pairing gap is possibly the largest one in Fig. 3(b), and others represent a new kind of mesoscopic effect which may or may not be related to superconductivity. Small $\delta$ of $\Delta_{\mathrm{P}}(\delta)$ does not match with any of pairing gaps calculated in Section 3, which means possibly that neither of the approximations
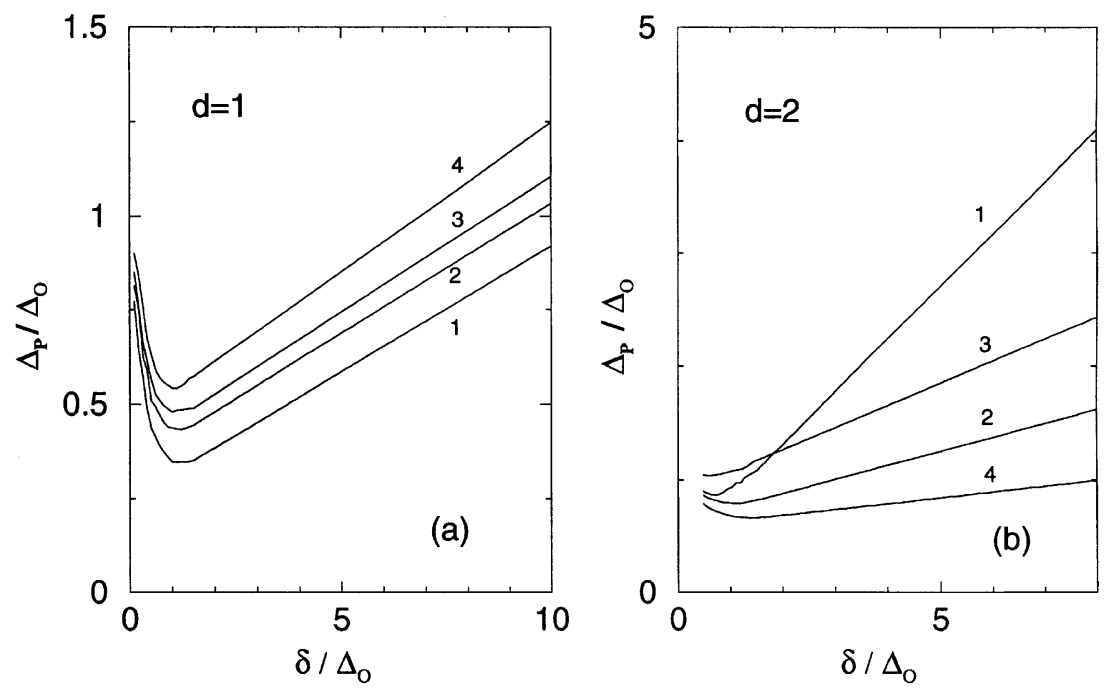

Fig. 3. Parity gap vs level spacing for non-degenerate case (a) and for double degenerate levels (b). In (a), numbers $1-4$ label dependences received with a number of levels in the interaction shell $n_{\mathrm{D}}=30,60,120$, and 360. In (b), numbers $1-4$ correspond to index $i$ in various pairing gaps defined according to $\Delta_{\mathrm{P}}^{(i)}=1 / 2\left(E_{4 m+i}+E_{4 m+i-2}\right)-E_{4 m+i-1}$. 

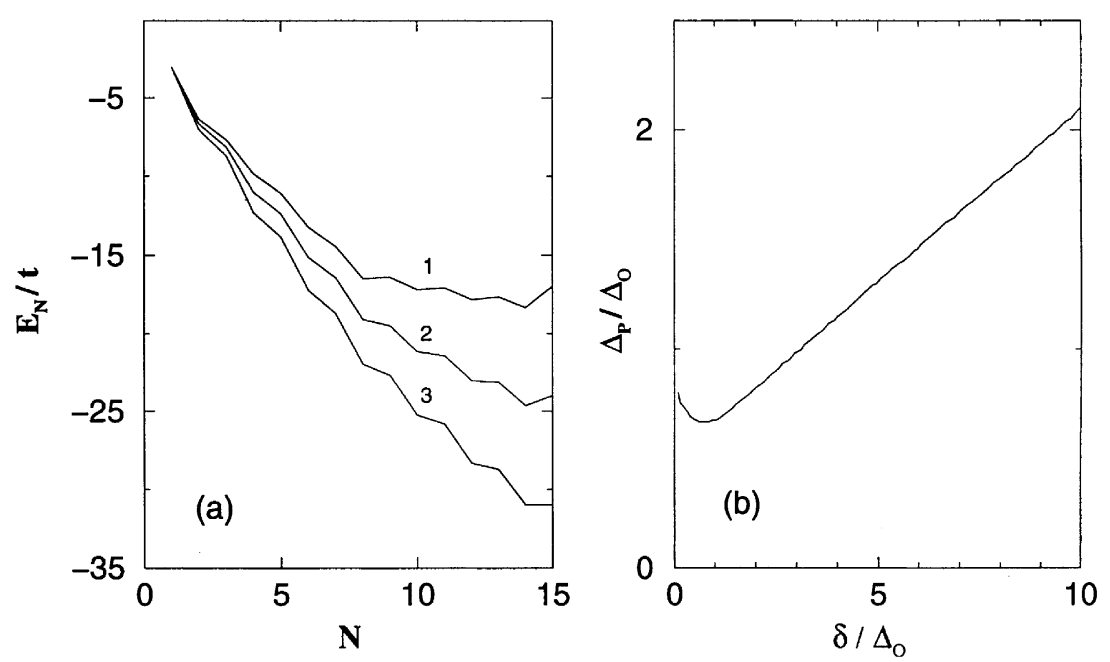

Fig. 4. (a) Energy vs number of electrons in cubic cluster with attractive interaction at sites. (1) $U / t=2$, (2) $U / t=3$, (3) $U / t=4$. (b) Parity gap vs level spacing in cubic cluster.

(original BCS or BCS with one level blocked) are good for small systems.

\section{Parity effect in atomic clusters}

We calculate the exact eigenstates of the negative-U Hubbard Hamiltonian

$$
\begin{aligned}
H= & -t \sum_{\langle i, j\rangle, \sigma} a_{i \sigma}^{\dagger} a_{j \sigma}-U \sum_{i=1}^{N} a_{i \uparrow}^{\dagger} a_{i \downarrow}^{\dagger} a_{i \downarrow} a_{i \uparrow} \\
& +\sum_{i \sigma} V_{i} a_{i \sigma}^{\dagger} a_{i \sigma}
\end{aligned}
$$

(with $U>0$ ) in small system of $N$ sites. $V_{i}$ is random potential at site $i$. The fact that Hamiltonian (4) commutes with the total number of electrons and also with the total spin leads to lowering of dimensionality $2^{2 N} \times 2^{2 N}$ of the matrix. Interaction is non-retarded (unlike BCS Hamiltonian) and momentum dependence of energy is non-parabolic. We do not believe however that these are crucial differences, and indeed the small- $U$ limit mean-field solution [13] corresponds to an energy gap $\Delta_{0} \simeq 12 t \exp (-12 t / U)$. Fig. 4a shows minimal energy $E_{N}$ of cubic cluster as a function of number of electrons $N$. Parity effect clearly, manifests itself in lower $E_{N}$ values for even $N$ and, when plotting $\Delta_{\mathrm{P}}$ against $\delta / \Delta_{0}$ (Fig. $4 \mathrm{~b}$ ), displays a behavior very similar to one received in the Richardson-Sherman model.

\section{Acknowledgements}

One of the authors (I.O.K) acknowledge helpful discussions with the participants of international Symposium on Mesoscopic Superconductivity (NTT, March 2000, Atsugi, Japan), especially with A.F. Andreev, H. Takayanagi and R.I. Shekhter. This work was partially supported by the Scientific and Technical Research Council of Turkey (TUBITAK) under grant no. TBAG 1736 and by the National Research Council of Italy, under the Research and Training Program for the Third Mediterranean Countries.

\section{References}

[1] P.W. Anderson, J. Phys. Chem. Solid 11 (1959) 28.

[2] C.T. Black, D.C. Ralph, M. Tinkham, Phys. Rev. Lett. 76 (1996) 688.

[3] D.C. Ralph, C.T. Black, J.M. Hengenrotter, J.G. Lu, M. Tinkham, in: L.L. Sohn, L.P. Kouwenhoven, G. Schön (Eds.), Mesoscopic Electron Transport, NATO ASI Series, vol. 345, Kluwer, Dordrecht, 1997, p. 447. 
[4] K.A. Matveev, A.I. Larkin, Phys. Rev. Lett. 78 (1997) 3749.

[5] F. Braun, J. von Delft, Phys. Rev. B 59 (1999) 9527.

[6] P. Ring, P. Shuck, The Nuclear Many-body Problem, Springer, New York, 1980.

[7] I.O. Kulik, I.K. Yanson, The Josephson Effect in Superconductive Tunneling Structures, Keter Press, Jerusalem, 1972 (Izdatel'stvo Nauka, Moscow, 1970).
[8] R.W. Richardson, N. Sherman, Nucl. Phys. 52 (1964) 221. [9] J. von Delft, F. Braun, cond-mat/9911058.

[10] H. Boyaci, Z. Gedik, I.O. Kulik, J. Superconductivity, in press.

[11] A. Mastellone, G. Falci, R. Fazio, Phys. Rev. Lett. 80 (1998) 4542.

[12] S.D. Berger, B.I. Halperin, Phys. Rev. B 58 (1998) 5213.

[13] I.O. Kulik, Physica 126B (1984) 280. 